\title{
Management of Pregnancy and Survival of Infants with Trisomy 13 or Trisomy 18
}

\author{
Sarah K. Dotters-Katz, MD ${ }^{1}$ Laura M. Carlson, MD ${ }^{1}$ Jasmine Johnson, MD ${ }^{1}$ Jacquelyn Patterson, MD ${ }^{2}$ \\ Matthew R. Grace, MD ${ }^{1}$ Wayne Price, MD ${ }^{2}$ Catherine J. Vladutiu, PhD ${ }^{1}$ Tracy A. Manuck, MD ${ }^{1}$ \\ Robert A. Strauss, $M D^{1}$
}

${ }^{1}$ Department of Obstetrics and Gynecology, University of North Carolina School of Medicine, Chapel Hill, North Carolina

2 Department of Pediatrics, University of North Carolina School of Medicine, Chapel Hill, North Carolina

\begin{abstract}
Address for correspondence Sarah K. Dotters-Katz, MD, Division of Maternal-Fetal Medicine, University of North Carolina School of Medicine, 3010 Old Clinic Building, CB \# 7516, Chapel Hill, NC 275997516 (e-mail: sarahdk@med.unc.edu).
\end{abstract}

Am J Perinatol 2016;33:1121-1127.

\begin{abstract}
\section{Keywords}

- trisomy 13

- trisomy 18

- antenatal testing

- neonatal intervention

- survival

Objective The objective of this study was to describe antenatal/intrapartum management and survival of liveborn infants with known trisomy 13 (T13) or trisomy 18 (T18) based on planned neonatal care.

Study Design This is a retrospective cohort study of singleton pregnancies complicated by T13/T18 at a tertiary center from 2004 to 2015. We included pregnancies with antenatal or neonatal cytogenetic T13/T18 diagnosis and excluded those which were terminated or had a fetal demise $<20$ weeks. We compared antenatal/intrapartum management and neonatal survival by planned neonatal care, defined as either neonatal intervention (INT), including neonatal cardiopulmonary resuscitative measures or comfort care (CC) without resuscitative measures.

Results In this study, 32 women (10 with T13 and 22 with T18) met study criteria; 12 (38\%) elected INT and 20 (62\%) CC. Compared with those who elected INT, women who elected CC were more likely to undergo elective induction ( 40 vs. $0 \%, p=0.01$ ), have an intrapartum stillbirth ( 0 vs. $32 \%, p=0.14$ ), and deliver vaginally ( 25 vs. $63 \%, p<0.01$ ). In neonatal survival analysis $(n=26)$, median survival was longer in the INT group compared with CC group (64 days [interquartile range, IQR: 2,155 ) vs. 3 days [IQR]: 0.3, 42 ), $p=0.28$ ), but survival to hospital discharge was similar (53 vs. $57 \%, p=0.95$ ).

Conclusion Regardless of desired level of neonatal INT, many women who continue pregnancies complicated by $\mathrm{T} 13 / 18$ have infants who survive beyond hospital discharge.
\end{abstract}

More women are delaying childbearing, and the percentage of women who became pregnant for the first time between the ages of 35 and 39 increased sixfold between 1973 and $2006 .^{1}$ There was a fourfold increase for women aged 40 to 44 years. ${ }^{1}$ Currently, approximately $15 \%$ of the pregnant population is older than 35 years. $^{2}$ As the risk of trisomy 13 (T13) and trisomy 18 (T18) is age related, more women are at risk of being diagnosed with fetal T13 or T18. ${ }^{3}$ Over the past 20 years, prenatal screening and diagnosis have improved dramatically through such technologies as ultrasound, maternal serum screening, and cell-free DNA screening. ${ }^{4}$ Though the overall incidence of T13 and T18 is low, 1:3,700 for T18 and 1:7,900 for $\mathrm{T} 13$, the rates of these are increasing due to the above factors. ${ }^{3}$

Outcomes and survival duration for infants born with T13 or T18 vary based on the degree of intervention (INT), as well as phenotype and genotype of the disorder. ${ }^{5-7}$ Counseling is further limited because existing data are often biased toward non-INT. ${ }^{8-10}$ Other data about the degree of INTs offered to and desired by the family are nonspecific. In addition, a provider's own bias may influence patient counseling. received

May 20, 2016

accepted

May 20, 2016

published online

July 20, 2016
Copyright $\odot 2016$ by Thieme Medical Publishers, Inc., 333 Seventh Avenue, New York, NY 10001, USA. Tel: +1(212) 584-4662.
DOI http://dx.doi.org/ 10.1055/s-0036-1585580. ISSN 0735-1631. 
Obstetric providers are further challenged by how to manage these pregnancies. Fetuses with T13 and T18 are often growth restricted and are known to be at very high risk for intrauterine fetal demise. ${ }^{11-13}$ There are no published data regarding antenatal and intrapartum management strategies for patients with a pregnancy complicated by T13 or T18.

The aim of this study was to describe antepartum and intrapartum management strategies for pregnancies complicated by $\mathrm{T} 13$ and $\mathrm{T} 18$ to provide more information for providers who are counseling families regarding these specific issues. In addition, we sought to compare survival duration for infants based on the degree of INT desired by the family.

\section{Materials and Methods}

We performed a retrospective cohort study of singleton pregnancies complicated by T13 or T18 at a tertiary referral center between January 2004 and April 2015. Patients were identified from the institution's cytogenetic database, the institution's perinatal database that catalogues all deliveries, and from the University of North Carolina Center for Maternal and Infant Health database. Identified cases were crossreferenced and duplicates were excluded.

Women with prenatally diagnosed or suspected fetal T13 or T18 who delivered at our institution were included in the study $(n=27)$. Infants who delivered at an outside institution and were transferred to our institution immediately after delivery were also included $(n=5)$. All women had diagnostic cytogenetic testing prenatally or immediately postpartum. Exclusion criteria included twin pregnancies, those who elected to terminate the pregnancy, those who experienced pregnancy loss before 20 weeks, and antepartum stillbirths. We planned to exclude mosaic infants; however, there were no cases of mosaicism in this cohort.

For analyses related to antepartum and intrapartum management, we included patients who had prenatal care and delivered at our institution $(n=27)$. For analyses related to survival, the five infants born at another institution and transferred to our neonatal intensive care unit immediately after delivery were included, but the six infants with intrapartum deaths from our center were not $(n=26)$. At our institution, when the diagnosis of T13 or T18 is made or strongly suspected, the family is given the choice of neonatal INT or comfort care (CC) at the time of delivery.

Chart review of both maternal and infant's records was performed by two physicians, the reviews were then spot checked by a third physician. Demographic data, ultrasound results, laboratory findings, counseling encounters, and pregnancy and delivery outcomes were abstracted from the maternal medical record. Infant survival and outcome data were obtained from the infant's medical record. Neonatal INT was defined as any INT in the delivery room or during the delivery admission. $\mathrm{CC}^{14}$ was defined as parental decision for no delivery room resuscitation or neonatal procedures (e.g., intubation). Families who wanted neonatal assessment at birth before deciding on INT or CC $(n=2)$ were classified based on delivery room decision. Indication for antenatal testing was defined based on the indication listed on the visit encounter form and included any of the following: nonstress test (NST), biophysical profile (BPP), umbilical artery Doppler studies, or growth ultrasound(s). Not all charts specified the degree of intrapartum monitoring. Fetal heart rate tracings were not available for most patients, thus continuous monitoring versus intermittent monitoring were determined based on intrapartum documentation.

Bivariate analysis was performed using Student $t$-test, Wilcoxon rank sum, and Kruskal-Wallis test for continuous variables and chi-square or Fisher exact test, as appropriate, for categorical variables. Antenatal and intrapartum management strategies were compared by parental desire for INT. Kaplan-Meier curves were performed for survival time based on parental desire for INT for all infants in the cohort as well as for T13 and T18 infants separately. Infants were followed until death or loss to follow-up; they were censored once they were lost to follow-up for $>12$ months. Log-rank tests were used to compare survival based on degree of INT and data were analyzed by an intention to treat analysis.

Statistical significance was defined as $p<0.05$. Stata 14.0 (StataCorp, College station, TX,) was used to perform the analysis. This study was approved by the University of North Carolina Institutional Review Board.

\section{Results}

During the 11-year study period, there were 64 cases with a cytogenetic diagnosis of T13 and 103 with T18. Of the T13 cases, 50 (78\%) elected termination and $4(6 \%)$ had an antepartum stillbirth and were thus excluded from this analysis. Among T18 cases, 77 (75\%) ended in termination and 4 (4\%) had an antepartum stillbirth. Overall, 10 fetuses with T13 and 22 fetuses with T18 met inclusion criteria for this analysis. Of these infants, 12 (38\%) parents elected for neonatal INT (T13: $n=5$; T18: $n=7)$, and $20(62 \%)$ parents planned for CC (T13: $n=5$; T18: $n=15$ ) (-Fig. 1).

Compared with women who desired CC, women who desired INT were older (38.5 vs. 30.0 years), and a higher percentage were primiparous ( - Table 1 ). No differences were observed by race/ethnicity, marital status, insurance status, and medical comorbidities. Women who selected INT were less likely to have a genetic counselor for a consultation (42 vs. 85\%) and less likely to have a prenatal diagnostic procedure such as amniocentesis or chorionic villous sampling (8.3 vs. $90 \%$ ).

When considering antenatal and delivery management, only 27 women who had prenatal care and delivered at our institution were included in the analysis. Of those women, 19 elected CC and 8 desired INT. All women had a targeted anatomy ultrasound, all of which were abnormal. Most women (89\%) had a maternal-fetal medicine consult (-Table 2). Over half of the women who desired CC had some form of antenatal testing; of these, more than $20 \%$ had BPPs and almost half had at least one ultrasound for fetal growth. In contrast, among those desiring INT, 88\% had at least one form of antenatal testing, 63\% had NSTs, 38\% had BPPs, and $88 \%$ had at least one growth ultrasound.

The median gestational age at delivery did not differ by desired INT (-Table 3 ). With regard to intrapartum fetal 


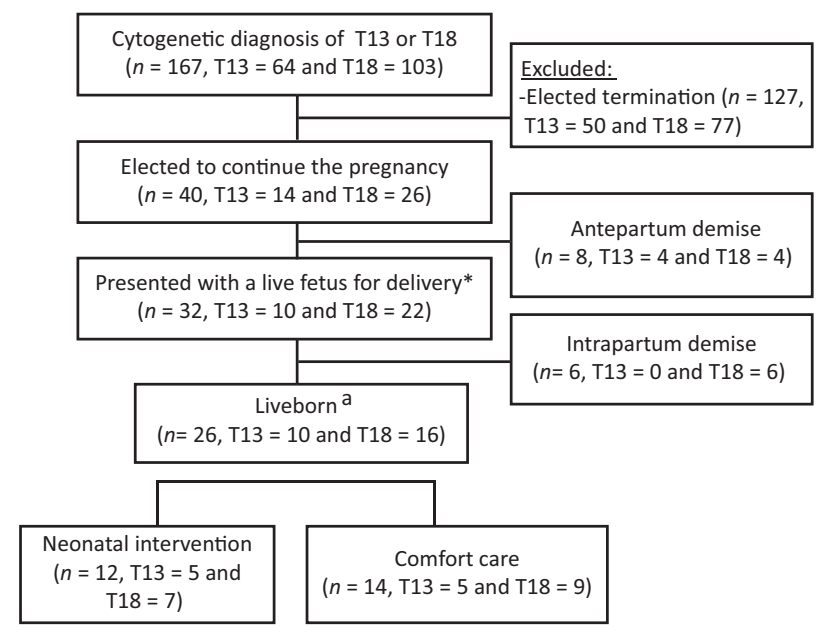

Fig. 1 Study population. Flow diagram documenting inclusion and exclusion criteria. This figure illustrates how patients came to be included in the study. The "*" refers to the fact of the 32 infants who survived to delivery, 5 infants were outborn, thus we did not have documentation antepartum or intrapartum monitoring. Therefore, only 27 infants were included in the antepartum and intrapartum analysis. The "a" denotes that only liveborn infants were included in the survival analysis.

monitoring, all women in the INT group and half of the women in the CC cohort had at least some form of monitoring (ranging from intermittent to continuous). There were six intrapartum deaths in the CC group: two who had intermittent monitoring and four where the degree of monitoring was
Table 2 Antepartum management strategies for women with a pregnancy complicated by T13 or T18 $(N=27)$, 2004-2015

\begin{tabular}{|l|l|l|l|}
\hline & $\begin{array}{l}\text { Intervention } \\
\boldsymbol{n}=\mathbf{8}(\%)\end{array}$ & $\begin{array}{l}\text { Comfort care } \\
\boldsymbol{n}=\mathbf{1 9}(\%)\end{array}$ & $p$-Value \\
\hline $\begin{array}{l}\text { Had perinatology } \\
\text { consultation }\end{array}$ & $6(75)$ & $18(95)$ & 0.20 \\
\hline $\begin{array}{l}\text { Had neonatology } \\
\text { consult }\end{array}$ & $2(25)$ & $10(53)$ & 0.24 \\
\hline $\begin{array}{l}\text { Any antenatal } \\
\text { testing }\end{array}$ & $7(88)$ & $10(53)$ & 0.19 \\
\hline Nonstress test & $5(63)$ & $2(11)$ & 0.01 \\
\hline $\begin{array}{l}\text { Biophysical } \\
\text { profile }\end{array}$ & $3(38)$ & $4(21)$ & 0.63 \\
\hline $\begin{array}{l}\text { Umbilical artery } \\
\text { Doppler }\end{array}$ & $2(25)$ & $2(11)$ & 0.56 \\
\hline $\begin{array}{l}\text { Growth } \\
\text { ultrasound }\end{array}$ & $7(88)$ & $9(47)$ & 0.09 \\
\hline
\end{tabular}

Abbreviations: T13, trisomy 13; T18, trisomy 18 .

ancludes nonstress test, biophysical profile, umbilical artery Doppler, or growth ultrasound.

not specified. There were no stillbirths in the INT group. Mode of delivery was different between the two groups. In the CC group, $89.5 \%$ of women delivered vaginally, with five of those being breech vaginal deliveries. In the CC group, there was one cesarean delivery for breech presentation and one for a

Table 1 Characteristics of women with a pregnancy complicated by T13 or T18 ( $N=32)$, 2004-2015

\begin{tabular}{|c|c|c|c|}
\hline & $\begin{array}{l}\text { Intervention } \\
n=12\end{array}$ & $\begin{array}{l}\text { Comfort care } \\
n=20\end{array}$ & $p$-Value \\
\hline Median maternal age (IQR) & $38.5(32.5,42.5)$ & $30(26.5,36.5)$ & 0.08 \\
\hline \multicolumn{4}{|l|}{ Insurance status (\%) } \\
\hline Private & $7(58)$ & $6(30)$ & \multirow[t]{3}{*}{0.31} \\
\hline Medicaid & $2(17)$ & $7(35)$ & \\
\hline Self-pay & $3(25)$ & $7(35)$ & \\
\hline \multicolumn{4}{|l|}{ Race $(\%)$} \\
\hline White & $3(25)$ & $7(35)$ & \multirow[t]{4}{*}{0.40} \\
\hline Black & $5(42)$ & $3(15)$ & \\
\hline Hispanic & $4(33)$ & $9(45)$ & \\
\hline Other & 0 & $1(3)$ & \\
\hline Married $(\%)^{a}$ & $9(90)$ & $16(89)$ & $>0.99$ \\
\hline Primiparous (\%) & $4(33)$ & $1(5)$ & 0.05 \\
\hline History of preterm delivery (\%) & $1(8)$ & 0 & 0.39 \\
\hline Any medical comorbidity $(\%)^{\mathrm{b}}$ & $3(25)$ & $2(10)$ & 0.34 \\
\hline Prenatal diagnosis (\%) ${ }^{c}$ & $1(8)$ & $18(90)$ & $<0.001$ \\
\hline Genetic counseling (\%) & $5(42)$ & $17(85)$ & 0.02 \\
\hline
\end{tabular}

Abbreviations: IQR, interquartile range; T13, trisomy 13; T18, trisomy 18 .

${ }^{a}$ Data only available for marital status on 28 women.

${ }^{\mathrm{b}}$ Medical issues included: type 1 or type 2 diabetes, chronic hypertension, or asthma.

cIncludes amniocentesis or chorionic villus sampling. 
1124 Management of Pregnancy and Survival of Infants with T13 or T18 Dotters-Katz et al.

Table 3 Intrapartum management strategies for women with a pregnancy complicated by T13 or T18 (N = 27), 2004-2015

\begin{tabular}{|l|l|l|l|}
\hline & $\begin{array}{l}\text { Intervention } \\
n=8\end{array}$ & $\begin{array}{l}\text { Comfort care only } \\
n=19\end{array}$ & $p$-Value \\
\hline Median GA at delivery (IQR) & $37(33.1,38.1)$ & $38(35.6,39.6)$ & 0.17 \\
\hline Elective induction (\%) & 0 & $8(40)$ & 0.01 \\
\hline Any monitoring (\%) & $8(100)$ & $10(53)$ & 0.06 \\
\hline Continuous monitoring (\%) & $5 / 7(71)$ & $4 / 10(40)$ & 0.07 \\
\hline Stillborn (\%) & 0 & $6(32)$ & 0.14 \\
\hline Mode of delivery (\%) & \multicolumn{3}{|l}{} \\
\hline Vaginal delivery & $2(25)$ & $12(63)$ & 0.003 \\
\hline Breech vaginal delivery & 0 & $5(26)$ & $2(11)$ \\
\hline Cesarean delivery & $6(75)$ & \\
\hline
\end{tabular}

Abbreviations: GA, gestational age; IQR, interquartile range; T13, trisomy 13; T18, trisomy 18 .

${ }^{\text {a }}$ The degree of monitoring was only documented in 17 cases: 7 in the intervention group and 10 in the comfort care group.

history of a prior cesarean delivery. In contrast, $75 \%$ of women in the INT group were delivered by cesarean. Indications for cesarean delivery included: nonreassuring fetal status $(n=2)$, arrest of dilation $(n=1)$, breech presentation $(n=1)$, prior cesarean $(n=1)$, and elective primary $(n=1)$.
Of the 32 women who presented with a live fetus for delivery, 6 (19\%) infants (all with T18) had intrapartum demise. Of those born alive $(n=26), 12$ had neonatal INT (T13: $n=5$; T18: $n=7$ ) and 14 had CC (T13: $n=5$; T18: $n=9$ )(-Table 4). All infants survived at least 1 hour; $11 / 12$ in

Table 4 Neonatal survival among infants with T13 or T18 $(N=26), 2004-2015$

\begin{tabular}{|c|c|c|c|}
\hline & Intervention & Comfort care & $p$-Value \\
\hline \multicolumn{4}{|l|}{ All patients: T13 or T18 $(n=26)$} \\
\hline $1 h^{a}(\%)$ & $12 / 12(100)$ & $14 / 14(100)$ & $>0.99$ \\
\hline $1 \mathrm{~d}^{\mathrm{a}}(\%)$ & $11 / 12(92)$ & $9 / 14(64)$ & 0.17 \\
\hline $1 \mathrm{wk}^{\mathrm{a}}(\%)$ & $9 / 12(75)$ & $5 / 14(36)$ & 0.045 \\
\hline $1 \mathrm{mo}^{\mathrm{a}}(\%)$ & $5 / 10(50)$ & $4 / 14(29)$ & 0.68 \\
\hline $1 \mathrm{y}^{\mathrm{a}}(\%)$ & $1 / 9(11)$ & $2 / 12(8.3)$ & $>0.99$ \\
\hline Median survival (IQR) & $64(2,155)$ & $3(0.5,42)$ & 0.28 \\
\hline Survival at hospital discharge (\%) & $7(58)$ & $8(5)$ & $>0.99$ \\
\hline \multicolumn{4}{|l|}{$\mathrm{T} 13(n=10)$} \\
\hline $1 \mathrm{~h}^{\mathrm{a}}(\%)$ & $5 / 5(100)$ & $5 / 5(100)$ & $>0.99$ \\
\hline $1 \mathrm{~d}^{\mathrm{a}}(\%)$ & $5 / 5(100)$ & $2 / 5(40)$ & 0.17 \\
\hline $1 w^{a}(\%)$ & $3 / 5(60)$ & $1 / 5(20)$ & 0.59 \\
\hline $1 \mathrm{mo}^{\mathrm{a}}(\%)$ & $1 / 4(25)$ & $1 / 5(20)$ & $>0.99$ \\
\hline $1 \mathrm{y}^{\mathrm{a}}(\%)$ & $1 / 4(25)$ & $0 / 4(0)$ & $>0.99$ \\
\hline Median survival (IQR) & $7.3(4,14)$ & $0.5(0.04,2)$ & 0.20 \\
\hline Survival at hospital discharge (\%) & $2(40)$ & $1(20)$ & $>0.99$ \\
\hline \multicolumn{4}{|l|}{$\mathrm{T} 18(n=16)$} \\
\hline $1 h^{\mathrm{a}}(\%)$ & $7 / 7(100)$ & $9 / 9(100)$ & $>0.99$ \\
\hline $1 \mathrm{~d}^{\mathrm{a}}(\%)$ & $6 / 7(86)$ & $7 / 9(78)$ & $>0.99$ \\
\hline $1 w^{a}(\%)$ & $6 / 7(86)$ & $4 / 9(44)$ & 0.15 \\
\hline $1 \mathrm{mo}^{\mathrm{a}}(\%)$ & $4 / 6(66)$ & $3 / 9(33)$ & 0.62 \\
\hline $1 \mathrm{y}^{\mathrm{a}}(\%)$ & $0 / 5(0)$ & $2 / 8(25)$ & 0.48 \\
\hline Median survival (IQR) & $150(19,155)$ & $22(3,40)$ & 0.85 \\
\hline Survival at hospital discharge (\%) & $5(71)$ & $7(78)$ & $>0.99$ \\
\hline
\end{tabular}

Abbreviations: IQR, interquartile range; T13, trisomy 13; T18, trisomy 18.

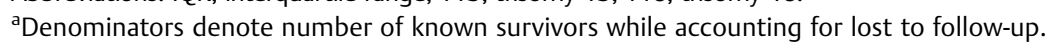




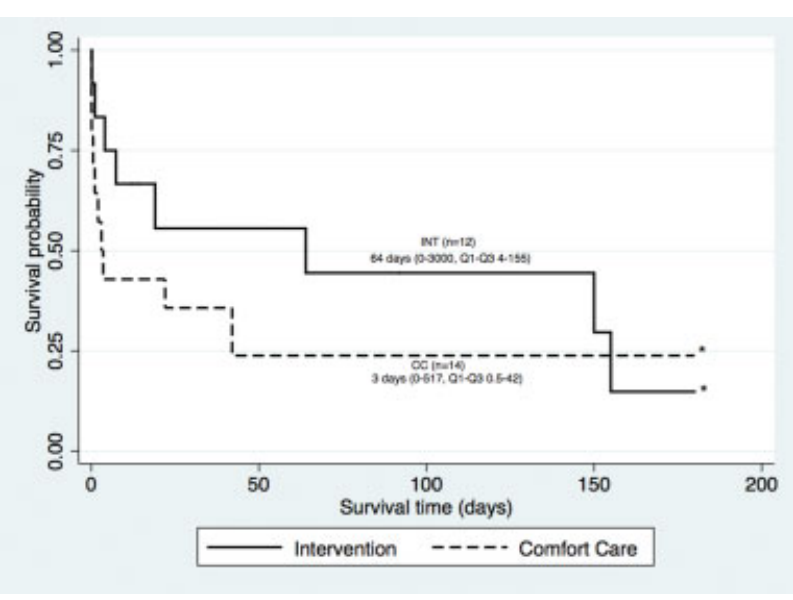

Fig. 2 Survival in liveborn T13/T18 based on type of care provided at delivery and in first 48 hours of life $(n=26) 2004$ to 2015. Kaplan-Meier curves showing survival stratified by CC $(n=14)$ compared with neonatal INT $(n=12)$. This figure compares neonatal survival for infants with T13 or T18 based on the degree of INT offered in the delivery room and first 48 hours of life. For those lost to follow-up, survival time was censored at the last documented time alive. There was no difference between the groups based on degree of INT. The "*” denotes 180 days of survival, a cutoff that was used to better accentuate early death. There was one alive at 180 days in the INT group and two in the CC group. Median survival time is listed in days, within the parenthesis is survival range, then interquartile range for survival denoted by Q1 to Q3. CC, comfort care; INT, intervention.

the INT group and 9/14 in the CC group survived at least 1 day and more than half of the infants in each group survived to hospital discharge. For infants receiving INT, median survival time after delivery was 64 days as compared with 3 days for those getting CC $(p=0.28)$ (-Figs. 2 and $\mathbf{3})$.

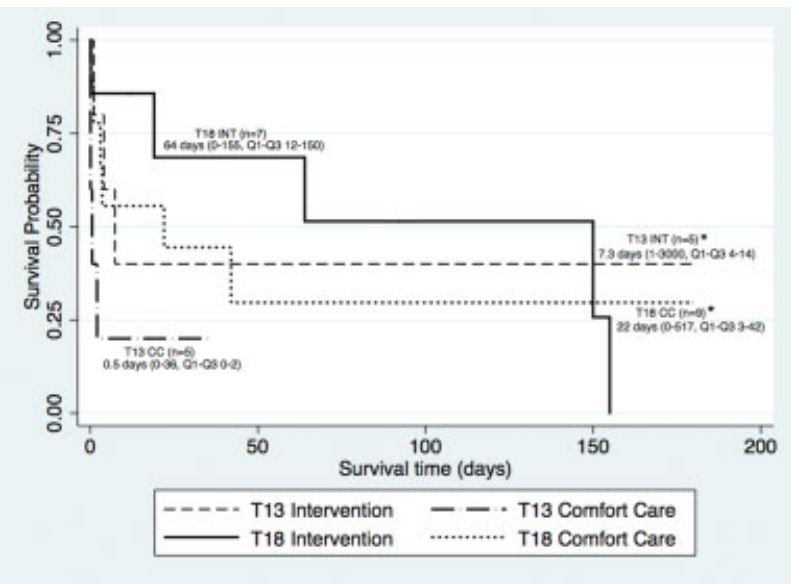

Fig. 3 Trisomy-specific survival by one type of care provided at delivery and in first 48 hours of life $(n=26) 2004$ to 2015. Kaplan-Meier curves showing survival stratified by T13 or T18, comparing INT to CC. This figure compares neonatal survival by trisomy type for infants based on the degree of INT offered in the delivery room and first 48 hours of life. For those lost to follow-up, survival time was censored at the last known time alive. There was no difference between the groups based on degree of INT. The “*” denotes 180 days of survival, a cutoff that was used to better accentuate early death. There was one alive at 180 days in the T13 INT group and two in the T18 CC group. Median survival time is listed in days, within the parenthesis is survival range, then interquartile range for survival denoted by $\mathrm{Q} 1$ to Q3. CC, comfort care; INT, intervention.

\section{Comment}

This study revealed that women with a pregnancy complicated by T13 or T18 who were planning for neonatal INT at delivery had more antenatal testing than those planning for $\mathrm{CC}$, although a large percentage of those planning for CC also had antenatal testing. A surprisingly large number of women in the CC group had continuous monitoring during labor. The mode of delivery was dramatically different between the two groups, with almost all CC fetuses delivering vaginally, even when they were in the breech presentation. Importantly, more than half of the infants in the CC group survived to hospital discharge. Based on this sample, infants with T13 or T18 who received neonatal INT survived longer.

There is a paucity of data regarding antepartum and intrapartum management of women with fetal T13 or T18. Due to the high rate of associated fetal anomalies and growth restriction, the issue of antenatal testing often arises for infants with $\mathrm{T} 13$ and T18. We were unable to identify any other published literature describing the use of antenatal testing in these fetuses. An unexpected finding was the significant rate of fetal testing among families who were planning for $\mathrm{CC}$. This testing was partly driven by growth ultrasounds, which also provided the family an opportunity to see the fetus additional times before delivery.

Intrapartum monitoring in the setting of a known diagnosis of T13 or T18 is also not well described in the literature. Cesarean delivery for fetal distress occurs frequently among infants where the diagnosis of T18 is unknown or suspected prenatally. ${ }^{15-17}$ Subramaniam et al estimated cesarean delivery rates of $42 \%$ for prenatally diagnosed and $69 \%$ for postnatally diagnosed T18 infants. ${ }^{7}$ Similar to our findings, other studies also suggest a high cesarean rate ( $>50 \%$ ), even in the setting of known T18. ${ }^{18,19}$ Interestingly, cesarean delivery rates appear to be lower among T13 fetuses in these prior studies. ${ }^{15,20}$ In the present study, we found cesarean delivery rates to be markedly higher for patients who desired neonatal INT when compared with those who desired only CC. Another significant finding in our study was the use of breech vaginal delivery to avoid cesarean delivery in women who desired CC only. When a provider is comfortable with breech deliveries, this should be considered over cesarean for women with a breech fetus with $\mathrm{T} 13$ or T18.

Many authors have described the natural history of T18. Recently, two large series including more than 1,100 infants with T18 described median survival of 7 to 8 days. ${ }^{21,22}$ These same studies examined similar outcomes for more than 800 infants with T13 and found median survival of 4.5 to 5 days. ${ }^{21,22}$ However, as these were both populationbased studies, the degree of neonatal INT was unable to be assessed. The question of the effects of aggressive compared with nonaggressive neonatal INT on survival was recently addressed by Subramaniam et al for neonates with T18. ${ }^{7}$ In that cohort of 46 infants with full T18, there were only 4 who had CC, compared with 42 who had any INT (of 
those 31 had aggressive INT and 11 had nonaggressive INT). When comparing the two INT groups, there was no difference in median survival. Given the small number of infants who did not receive any INT, that group was not analyzed independently and was excluded from the analysis. However, similar to our study, the authors found that long-term survival was rare, with only two living more than 1 year. ${ }^{7}$ It should be noted that for one of the infants in the CC group, after surviving for more than 2 months at home, the parents requested pediatric INTs. This infant was included in the CC group based on our intention to treat analysis. However, the outcomes at more than 2 months could be due to INT.

Our study is the first to specifically compare CC only with neonatal INT with respect to neonatal survival. Providers often counsel patients that duration of survival is short for these infants. One original finding in this study was that even with CC, over half of the infants survived to go home with their families. This raises an important issue when counseling families and when caring for families after delivery. Setting the expectation that their infant may go home with them and may die at home is important. Ensuring that the appropriate resources (such as neonatal hospice) exist and are in place before discharge to help a family in this situation is essential. To accurately counsel a family with fetal T13 or T18, an understanding of survival duration based on degree of INT is important.

This study is limited by a small sample size, and thus may not be powered to show differences in outcomes. The data are also from a single center, and provider attitudes may be biased. However, due to the long time period over which data were collected, there were several providers with varying attitudes toward management of these pregnancies, limiting this bias. Since this is a tertiary referral center, we were also limited in that some patients traveled far to deliver and many of them were lost to follow-up after discharge. Finally, due to the retrospective nature of the study, not all data points were available for all subjects.

Despite these limitations, this study adds to the literature regarding antepartum and intrapartum management of T13 and T18. The realization that many families who desire CC at delivery are concerned regarding fetal wellbeing in utero and thus may receive antenatal testing is important for providers to consider. These concerns may extend to a desire for intrapartum monitoring for patients who desire CC as well. Many neonates only receiving CC survive to leave the hospital with their family and may survive for days to weeks or longer. All of these details are important to consider when counseling at the time of diagnosis and during prenatal care for women who continue to the pregnancy.

\section{Note}

This material was presented in poster format at the Society for Maternal-Fetal Medicine's Pregnancy Meeting, Atlanta, Georgia, February 2016.

\section{Funding}

None.

\section{Conflict of Interest}

None.

\section{References}

1 Mathews TJ, Hamilton BE. First births to older women continue to rise. NCHS Data Brief 2014;(152):1-8

2 Martin JA, Hamilton BE, Ventura SJ, Osterman MJ, Mathews TJ. Births: final data for 2011. National vital statistics reports: from the Centers for Disease Control and Prevention, National Center for Health Statistics. Natl Vital Stat Rep 2013;62(1): 1-69

3 Mai CT, Kucik JE, Isenburg J, et al; National Birth Defects Prevention Network. Selected birth defects data from population-based birth defects surveillance programs in the United States, 2006 to 2010: featuring trisomy conditions. Birth Defects Res A Clin Mol Teratol 2013;97(11):709-725

4 Irving C, Richmond S, Wren C, Longster C, Embleton ND. Changes in fetal prevalence and outcome for trisomies 13 and 18: a population-based study over 23 years. J Matern Fetal Neonatal Med 2011;24(1):137-141

5 Lin HY, Lin SP, Chen YJ, et al. Clinical characteristics and survival of trisomy 18 in a medical center in Taipei, 1988-2004. Am J Med Genet A 2006;140(9):945-951

6 Maeda J, Yamagishi H, Furutani Y, et al. The impact of cardiac surgery in patients with trisomy 18 and trisomy 13 in Japan. Am J Med Genet A 2011;155A(11):2641-2646

7 Subramaniam A, Jacobs AP, Tang Y, et al. Trisomy 18: a singlecenter evaluation of management trends and experience with aggressive obstetric or neonatal intervention. Am J Med Genet A 2016;170(4):838-846

8 Guon J, Wilfond BS, Farlow B, Brazg T, Janvier A. Our children are not a diagnosis: the experience of parents who continue their pregnancy after a prenatal diagnosis of trisomy 13 or 18 . Am J Med Genet A 2014;164A(2):308-318

9 Niedrist D, Riegel M, Achermann J, Schinzel A. Survival with trisomy 18-data from Switzerland. Am J Med Genet A 2006; 140(9):952-959

$10 \mathrm{Wu}$ J, Springett A, Morris JK. Survival of trisomy 18 (Edwards syndrome) and trisomy 13 (Patau syndrome) in England and Wales: 2004-2011. Am J Med Genet A 2013;161A(10):2512-2518

11 Burke AL, Field K, Morrison JJ. Natural history of fetal trisomy 18 after prenatal diagnosis. Arch Dis Child Fetal Neonatal Ed 2013; 98(2):F152-F154

12 Lakovschek IC, Streubel B, Ulm B. Natural outcome of trisomy 13, trisomy 18 , and triploidy after prenatal diagnosis. Am J Med Genet A 2011;155A(11):2626-2633

13 Morris JK, Savva GM. The risk of fetal loss following a prenatal diagnosis of trisomy 13 or trisomy 18. Am J Med Genet A 2008; 146A(7):827-832

14 Vonnahme KA, Hess BW, Hansen TR, et al. Maternal undernutrition from early- to mid-gestation leads to growth retardation, cardiac ventricular hypertrophy, and increased liver weight in the fetal sheep. Biol Reprod 2003;69(1):133-140

15 Houlihan OA, O'Donoghue K. The natural history of pregnancies with a diagnosis of trisomy 18 or trisomy 13; a retrospective case series. BMC Pregnancy Childbirth 2013;13:209

16 Rosa RF, Rosa RC, Lorenzen MB, et al. Trisomy 18: experience of a reference hospital from the south of Brazil. Am J Med Genet A 2011;155A(7):1529-1535

17 Embleton ND, Wyllie JP, Wright MJ, Burn J, Hunter S. Natural history of trisomy 18. Arch Dis Child Fetal Neonatal Ed 1996;75(1): F38-F41 
18 David TJ, Glew S. Morbidity of trisomy 18 includes delivery by caesarean section. Lancet 1980;2(8207):1295

19 Young ID, Cook JP, Mehta L. Changing demography of trisomy 18. Arch Dis Child 1986;61(10):1035-1036

20 Barry SC, Walsh CA, Burke AL, McParland P, McAuliffe FM, Morrison JJ. Natural history of fetal trisomy 13 after prenatal diagnosis. Am J Med Genet A 2015;167A(1):147-150
21 Meyer RE, Liu G, Gilboa SM, et al; National Birth Defects Prevention Network. Survival of children with trisomy 13 and trisomy 18: a multi-state population-based study. Am J Med Genet A 2016; 170(4):825-837

22 Vendola C, Canfield M, Daiger SP, et al. Survival of Texas infants born with trisomies 21, 18, and 13. Am J Med Genet A 2010; 152A(2):360-366 